\title{
Stoffwechselveränderungen und Ernährungstherapie von Patienten nach großen viszeralchirurgischen Eingriffen und bei chirurgischen Intensivpatienten
}

\author{
Wolfgang H. Hartl ${ }^{\mathrm{a}}$ Elke Muhl ${ }^{\mathrm{b}}$ \\ ${ }^{a}$ Chirurgische Klinik und Poliklinik der Universität, Campus Grosshadern, Ludwig-Maximilians-Universität, München, \\ ${ }^{\mathrm{b}}$ Klinik für Chirurgie, Universitätsklinikum Schleswig-Holstein, Campus Lübeck, Deutschland
}

Schlüsselwörter

Enterale Ernährung · Parenterale Ernährung ·

Chirurgische Intensivstation

\section{Zusammenfassung}

Nach chirurgischer Homöostasestörung kommt es durch hormonelle sowie immunologische Veränderungen regelhaft zu charakteristischen passageren Stoffwechselveränderungen (Hyperglykämie, Eiweißkatabolie), die zwar entwicklungsgeschichtlich sinnvoll waren, in Zeiten der modernen Medizin aber kontraproduktiv sind. Ziel der perioperativen Ernährungstherapie ist es somit, sekundäre metabolische Schäden soweit wie möglich zu begrenzen und damit die Prognose des Patienten zu verbessern. Eckpfeiler jeder Ernährungstherapie ist die Zufuhr von ausreichend Eiweiß oder Aminosäuren (in der Regel 1,2-1,5 g/kg/Tag) und die Verhinderung von Hyperglykämien (>180 mg/dl) durch die reduzierte Zufuhr von Kohlenhydraten in der postoperativen Akutphase. Als Ernährungsmodus sollte dabei die orale bzw. enterale Substratzufuhr immer bevorzugt werden. Voraussetzung ist jedoch ein funktionstüchtiger oberer und unterer Gastrointestinaltrakt, der somit engmaschig im Hinblick auf Passagestörungen, aber auch Malabsorption zu überwachen ist. Die Quantität und Qualität der oralen/enteralen postoperativen Ernährung sollte dabei die Besonderheiten des chirurgischen Eingriffs berücksichtigen. Bei bestimmten Patientengruppen (onkologische Patienten) ist bereits eine präoperative Konditionierung mittels einer speziellen Immunonutrition sinnvoll. Nur bei gastrointestinaler Dysfunktion in Verbindung mit einer präoperativ bereits bestehenden Mangelernährung ist postoperativ eine parenterale Ernährung indiziert. Mangelernährte Patienten können präoperativ durch klinische Untersuchung (Subjective Global Assessment) identifiziert werden. Unter parenteraler Ernährung ist eine besonders intensive Überwachung im Hinblick auf Blutzucker-, Triglyzerid- und Elektrolytkonzentrationen nötig. Dabei ist bei Intensivpatienten die zusätzliche Zufuhr von Glutamin sinnvoll. Eine parenterale Fettapplikation ist nur unter Langzeittherapie erforderlich.
Keywords

Enteral nutrition - Parenteral nutrition .

Surgical Intensive Care Unit

\section{Summary}

Changes of Metabolism and Nutrition Therapy in Patients with Major Visceral Surgical Interventions and in Surgical Intensive Care Patients

Surgical injury results in a variety of hormonal and immunologic reactions causing characteristic temporary metabolic changes (hyperglycemia, muscle protein catabolism). Although useful during the dawn of mankind, these metabolic changes are counterproductive in times of modern medicine. Perioperative nutrition tends to limit such secondary metabolic complications as much as possible, thereby improving patient prognosis. The cornerstone of each nutritional therapy is the supplementation of sufficient amounts of protein or amino acids (1.2-1.5 g/kg/day). Furthermore, hyperglycemia ( $>180 \mathrm{mg} / \mathrm{dl}$ ) should be prevented by reducing the provision of carbohydrates during the postoperative acute phase. Oral/ enteral nutrition should always be the application mode of choice. It is essential, however, that the upper and lower gastrointestinal tract is functioning properly. Therefore, a close surveillance regarding a potential deterioration of motility as well as absorption is mandatory. Quantity and quality of oral/ enteral foods depends on the particularities of the surgical procedure. Patients with malignant diseases will profit from a preoperative nutritional conditioning (immunonutrition). Only patients with gastrointestinal dysfunction, who are simultaneously malnourished, benefit from postoperative parenteral nutrition. Malnutrition can be identified preoperatively by subjective global assessment. During parenteral nutrition, it is particularly important to closely monitor concentrations of blood glucose, triglycerides, and electrolytes. In critically ill patients, additional glutamine should be provided during all periods of parenteral substrate supply, whereas supplementation of intravenous fat is restricted to patients requiring a prolonged parenteral nutrition.

\section{KARGER}

Fax +497614520714

Information@Karger.de

www.karger.com
(C) 2011 S. Karger GmbH, Freiburg

$1662-6664 / 11 / 0271-0028 \$ 38.00 / 0$

Accessible online at:

www.karger.com/vim
Prof. Dr. Elke Muhl

Klinik für Chirurgie, Universitätsklinikum Schleswig-Holstein Campus Lübeck

Ratzeburger Allee 160, 23538 Lübeck, Deutschland

Tel. +49 451 500-2025, Fax -2026

Elke.muhl@uk-sh.de 


\section{Einleitung}

Die Betreuung chirurgischer Patienten umfasst in der Regel zwei große Patientengruppen:

1. Patienten, die präoperativ bis auf ihre Grunderkrankung gesund sind und die postoperativ einen regelhaften Verlauf aufweisen;

2. Patienten mit prä- bzw. postoperativen Organfunktionsstörungen (in der chirurgischen Intensivmedizin in der Regel hervorgerufen durch SIRS (systemisches inflammatorisches Response-Syndrom) oder Sepsis) und Patienten mit drohenden oder bereits vorbestehenden Mangelernährungszuständen, die intensiv therapiert werden müssen. Die Ernährungstherapie ist ein wichtiger Bestandteil der Therapie beider Kollektive.

Vor allem um die letztere Patientengruppe, die Risikopatienten, soll es im Folgenden gehen. Die Kenntnis der pathophysiologischen Veränderungen bei diesen Patienten ist Grundvoraussetzung für eine im Folgenden darzustellende leitliniengerechte und patientenadaptierte Ernährungstherapie.

\section{Postaggressionsstoffwechsel}

Die Antwort des Patienten auf eine Homöostasestörung (Operation, Verletzung, SIRS, Sepsis) ist durch unterschiedlichste endokrine, metabolische und immunologische Veränderungen charakterisiert. Diese Veränderungen haben prognostische Bedeutung für den Heilungsverlauf.

Es kommt einerseits zu einer Aktivierung des unspezifischen Immunsystems (systemische Hyperinflammation) und zum zweiten zur Ausbildung des sog. Postaggressionssyndroms. Diese beiden Phänomene können in Abhängigkeit vom Ausmaß ihrer Ausprägung und von der Zeitdauer unter Umständen zu Organversagen führen.

In Verbindung mit dem Postaggressionssyndrom beobachtet man eine progrediente Eiweißkatabolie mit weitreichenden Folgen für Wundheilung, Organversagen, Inzidenz nosokomialer Infektionen usw. Die Ernährungstherapie kann hier einen wichtigen Beitrag in der Behandlung viszeralchirurgischer Patienten leisten, auch wenn sie nicht in der Lage ist, die neuronalen und humoralen Veränderungen im Postaggressionsstoffwechsel zu verhindern [1-3].

Eine chirurgische Homöostasestörung ist durch die Freisetzung örtlicher bioaktiver Substanzen (z.B. Cytokine), gesteigerte neuronale (z.B. Schmerz) und systemische Reaktionen (Tachykardie und Hypertension) und eine Aktivierung von $\beta$-Rezeptoren mit extravasalen Volumenverschiebungen charakterisiert.

\section{Sympathische Achse}

Zur Regulierung physiologischer Vorgänge besitzt der Hypothalamus zwei bedeutende Efferenzen. Die eine besteht in der sympathischen Achse, zusammengesetzt aus dem Nebennie- renmark (Adrenalin- und Noradrenalinfreisetzung) und dem sympathischen Nervensystem. Die zweite, wesentliche Efferenz beinhaltet die Hypothalamus-Hypophysenachse [4].

Katecholamine tragen wesentlich zum Anstieg des Energieumsatzes bei, der nach chirurgischer Homöostasestörung $\mathrm{zu}$ beobachten ist, und bewirken zusammen mit anderen Stresshormonen eine Umstellung des Kohlenhydrat- und Eiweißstoffwechsels. So beschleunigt Adrenalin die hepatische Glykogenolyse und Glukoneogenese. Ferner mobilisieren Katecholamine freie Fettsäuren durch einen direkten Effekt aus dem Fettgewebe und auch sekundär über die Unterdrückung der pankreatischen Insulinfreisetzung. Da sowohl $\alpha$ - als auch $\beta$-adrenerge Rezeptoren auf den Betazellen des pankreatischen Apparates vorhanden sind, sind entsprechend den Katecholeminkonzentrationen sowohl inhibierende $(\alpha$-adrenerge) als auch stimulierende ( $\beta$-adrenerge) Auswirkungen auf die Insulinfreisetzung möglich. Bei zunehmendem Schweregrad der Homöostasestörung und Überwiegen der $\alpha$-Wirkung wird somit die pankreatische Insulinfreisetzung gehemmt. Die Freisetzung von Glukagon aus pankreatischen $\alpha$-Zellen wird durch $\beta$-adrenerge Rezeptoren stimuliert und ist dadurch im Rahmen der Stressreaktionen deutlich gesteigert.

Unter normalen Umständen ist die Glukosekonzentration der wichtigste Stimulus für die pankreatische Insulinfreisetzung [5]. Insulin besitzt eine globale anabolische Wirkung und steigert die muskuläre Proteinsynthese, die hepatische Glykogensynthese und die Glykolyse. Gleichzeitig wird der Glukosetransport in die Zelle hinein sowie die Lipogenese im Fettgewebe und die Proteinsynthese in zahlreichen Kompartimenten beschleunigt. Nach chirurgischer Homöostasestörung kann man zwei verschiedene Phasen der Insulinfreisetzung beobachten. Die erste Phase spielt sich innerhalb der ersten Stunden nach einem chirurgischen Trauma ab und manifestiert sich als eine relative Unterdrückung der Insulinfreisetzung; ein Phänomen, das ganz überwiegend den Einfluss der Katecholamine und die Aktivierung des sympathischen Nervensystems reflektiert. In einer späteren Phase kommt es dann zu einer Normalisierung bzw. zu einem leichten Anstieg der Insulinkonzentrationen, der jedoch dem Ausmaß der gleichzeitig zu beobachtenden Hyperglykämie nicht entspricht. Wir sehen dann das Bild der sog. peripheren Insulinresistenz, das durch das Überwiegen der antiinsulinären Hormone (Katecholamine, Glukagon, Kortison) entsteht.

Glukagon hat im Gegensatz zu Insulin katabole Eigenschaften; es hemmt die Proteinsynthese und stimuliert die hepatische Glykogenolyse und Glukoneogenese. Der synergistische Effekt der Katecholamine, des Glukagons und der Glukokortikoide auf die hepatische Glukoneogenese ist dafür verantwortlich, dass dieser Stoffwechselweg bei gleichzeitiger Erhöhung aller drei Stresshormonkonzentrationen nach chirurgischem Trauma maximal gesteigert ist und bei entsprechend konstanter Hormonaktivierung auch über längere Zeiträume beschleunigt bleiben kann. 


\section{Hypothalamus-Hypophysenachse}

Nach chirurgischer Homöostasestörung kommt es innerhalb von Minuten zu einer Aktivierung der adrenokortikalen Achse mit rapidem Anstieg von Corticotropin-freisetzenden Hormonen $(\mathrm{CRH})$, adrenocorticotropen Hormonen (ACTH) und Glukokortikoiden um ein Vielfaches über den Ausgangswert.

Glukokortikoide stimulieren die hepatische Glukoneogenese und steigern die Speicherung von Kohlenhydraten in Form von Glykogen in der Leber; gleichzeitig wird die Insulinempfindlichkeit im gesamten Organismus verringert. Kortisol ist ein kataboles Hormon und setzt über eine Steigerung der Proteinabbaurate und Hemmung der Proteinsynthese Aminosäuren aus extrahepatischen Geweben frei. Kortisol steigert ferner die Mobilisierung von freien Fettsäuren aus dem Fettgewebe und erhöht damit die Konzentration der freien Fettsäuren im Plasma. Durch die vermehrte Verfügbarkeit von freien Fettsäuren wird somit bei fehlender exogener Nährstoffzufuhr der Energiebedarf überwiegend durch Oxidation von Fettsäuren gedeckt. Die Kohlenhydratutilistaion bleibt obligat zuckerabhängigen Geweben vorbehalten.

\section{Weitere endokrine Besonderheiten des Intensivpatienten}

Die Aktivität der sympatoadrenalen Achse bzw. die pankreatische Insulin- und Glukagonfreisetzung korrelieren eng mit dem Krankheitsschweregrad und der Ausprägung des SIRS bzw. der Sepsis auch über längere Zeit. Im Gegensatz dazu zeigt die Aktivierung der Hypothalamus-Hypophysenachse eine deutliche zeitliche Abhängigkeit, die nicht unbedingt mit dem klinischen Krankheitsschweregrad übereinstimmt [4].

Bei bis zu 50\% der Patienten mit protrahierter Sepsis/ Mehrfachorganversagen kann eine Insuffizienz der adrenokortikalen Achse gefunden werden, mit inadäquatem Kortisolanstieg nach Stimulation mit exogenem ACTH. Gleichzeitig sind die Konzentrationen des endogenen ACTH im Normalbereich.

Ebenfalls betroffen ist die thyreoidale Achse. Es findet sich bei Intensivpatienten praktisch regelhaft eine ausgeprägte biochemische Hypothyreose, gekennzeichnet durch niedrige $\mathrm{T}_{4}$ - bzw. $\mathrm{T}_{3}$-Konzentrationen. Inwieweit es sich bei den Veränderungen der thyreoidalen Achse um ein Epiphänomen oder um ein echtes Defizit handelt, das den Krankheitsverlauf ungünstig beeinflusst, ist immer noch umstritten.

Als weiterer wesentlicher Befund imponiert ein Abfall der Wachstumshormonkonzentration. Dieser relative Hyposomatotropismus bei länger anhaltendem Mehrfachorganversagen unterstützt die gleichzeitig immer zu beobachtende ausgeprägte Eiweißkatabolie.

\section{Veränderungen des Substratstoffwechsels nach chirurgischer}

Homöostasestörung sowie bei SIRS und Sepsis

Die umschriebenen hormonellen Veränderungen stellen die wesentliche Basis für die Umstellung des Substratstoffwechsels nach chirurgischem Trauma dar. Hierbei steht die Katabolie aller im Körper vorhandenen Substratdepots im Mittel- punkt. So kommt es im Fettgewebe zu einer gesteigerten Lipolyse mit vermehrter Freisetzung von freien Fettsäuren.

Parallel zur eingeschränkten Kohlenhydratverwertung im Skelettmuskel kommt es zu einem ausgeprägten Eiweißabbau. Die so freigesetzten Aminosäuren dienen im Wesentlichen in der Leber zur beschleunigten Neuproduktion von Glukose, und sie sind essenziell für die beschleunigte hepatische Proteinsynthese und für die Wundheilung. In diesem Zusammenhang ist auch die Versorgung des Gastrointestinaltrakts mit bestimmten Aminosäuren (Glutamin) von Bedeutung.

Aus Glukoneogenese und Glykogenolyse werden vermehrt Kohlenhydrate ins Blut abgegeben. Die beschleunigte hepatische Glukoseproduktion erzeugt zusammen mit der peripheren Insulinresistenz eine Hyperglykämie.

In der Leber werden Akutphasenproteine gebildet (z.B. Creaktives Protein (CRP)), die eine wichtige Rolle bei der Überwindung der traumainduzierten Homöostasestörung spielen. Diese Proteine besitzen ausgeprägte antiinflammatorische Eigenschaften.

Die gesteigerte Glukoneogenese führt zum Verlust von Harnstoff-Stickstoff und ist das biochemische Korrelat für die Abnahme von Muskelmasse.

Die einzelnen Stoffwechselveränderungen erleben ihren Peak jedoch nicht zum gleichen Zeitpunkt. Die Insulinresistenz mit begleitender Hyperglykämie ist bereits in den ersten $48 \mathrm{~h}$ maximal ausgeprägt, wohingegen die Abnahme des Körpereiweißbestandes erst nach 2 Wochen ihr Maximum erreicht. Erst 3-6 Monate nach komplikationslosem chirurgischem Trauma kann mit einer Wiederauffüllung des Körpereiweißbestandes gerechnet werden.

Bei persistierend schwerem SIRS bzw. schwerer Sepsis bleiben aufgrund der anhaltenden hormonellen Aktivierung auch die schwere Störung des Kohlenhydratstoffwechsels mit Hyperglykämie und Insulinresistenz und die Eiweißkatabolie erhalten.

Eine sog. septische Neuropathie findet sich bei 70-90\% aller Intensivpatienten mit peripherer Polyneuropathie und Zeichen einer axonalen Degeneration. In der Folge kommt es so zu einer funktionellen Denervierung und damit auch zu charakteristischen Veränderungen im Eiweißstoffwechsel des nachgeschalteten Organs, d.h. des peripheren Muskels. Letzterer reagiert auf eine derartige Denervierung mit einer drastischen Erhöhung der Proteinabbaurate. Über 90\% der kritisch kranken Patienten zeigen eine Atrophie des Skelettmuskels.

Ein weiterer zentraler Befund bei fast allen Intensivpatienten ist die zunehmende Leberverfettung, die aus einem Ungleichgewicht zwischen Fettsäureaufnahme, Fettsäureoxidation und Fettsäuregabe über die VLDL(Very Low Density Lipoprotein)-Triglyzeride resultiert.

\section{Ziele der Ernährungstherapie}

Ziel einer Ernährungstherapie unserer Patienten muss sein, die Auswirkungen der Homöostasestörung durch die Opera- 
Abb. 1. Variablen für das «Subjective Global Assessment» (SGA).
A. Anamnese

1. Gewichtsveränderung

Gesamtverlust in den letzten 6 Monaten: Menge $=\#$

prozentualer Verlust $=\#$

Veränderungen in den letzten 2 Wochen: $\mathrm{kg}$;

2. Veränderungen in der Nahrungszufuhr (bezogen auf den Normalzustand) Zunahme unverändert

\begin{tabular}{ll}
\hline & unverändert \\
& verändert \\
\hline Art der Veränderung:
\end{tabular}

Dauer $=$ \# Wochen hypokalorische feste Nahrung, komplette flüssige Diät hypokalorische flüssige Diät, Fasten

3. Gastrointestinale Beschwerden (länger als 2 Wochen anhaltend)

4. Körperliche Aktivität $\begin{array}{lr}\text { keine; } & \text { Übelkeit; } \\ \text { Durchfall; __ Anorexie }\end{array}$ keine Einschränkungen (z.B. voll arbeitsfähig) eingeschränk Art der Einschränkung: Dauer = \# Wochen leicht reduzierte Belastbarkeit geringe Belastbarkeit

5. Grunderkrankung und ihr Verhältnis zum Ernährungsbedarf Hauptdiagnose Energiebedarf (Stress): kein Stress geringer Stress mäßiger Stress starker Stress

B. Körperlicher Untersuchungsbefund (für jede Variable ist zu spezifizieren in $0=$ normal, $1+=$ gering, $2+=$ mäßig, $3+=$ stark)

\#_ Verlust von subkutanem Fett (über M. trizeps, Brustkorb)

\#—Muskelschwund (M. quadriceps, deltoideus)

\#— Knöchelödeme

Flankenödeme

\#_Aszites

C. SGA-Klassifizierung

$A=$ gut ernährt

$B=$ mäßige Mangelernährung (auch fraglich)

$\mathrm{C}=$ schwere Mangelernährung

Die gefundenen Kriterien sind anzukreuzen bzw. bei \# sind die entsprechenden numerischen Werte einzugeben. tion so gering wie möglich zu halten, Komplikationen zu vermeiden und so den Heilungsverlauf zu unterstützen und $\mathrm{zu}$ beschleunigen. Im Einzelnen soll das Ausmaß der Katabolie möglichst minimiert werden. Selbst bei nicht intensivbehandlungspflichtigen Patienten ist eine nicht ausreichende Nahrungszufuhr für mehr als 14 Tage mit einer erhöhten Letalität assoziiert. Präoperative Mangelernährungszustände haben eine erhöhte Morbidität zur Folge und vergrößern das Ausmaß und die Folgen der oben beschriebenen postoperativen metabolischen Veränderungen [6]. Wann immer möglich, sollten sie präoperativ ausgeglichen werden und gegebenenfalls bei der postoperativen Ernährung Berücksichtigung finden.

\section{Einschätzung des Ernährungszustands}

Aus klinischer Sicht hat sich die Einteilung in normal ernährt, mäßig mangelernährt oder schwer mangelernährt bewährt. Als mäßig mangelernährt gelten Patienten mit einem Körpergewichtsverlust von 10-15\% vor dem Intensivaufenthalt und einer gleichzeitigen Hypalbuminämie bzw. Störung in anderen Organsystemen. Eine schwere Mangelernährung besteht bei einem Gewichtsverlust von $15 \%$ oder mehr. Für die Praxis hat sich die Einschätzung des Ernährungszustands anhand des sog. «Subjective Global Assessment (SGA)» durchgesetzt. Diese Methode beruht im Wesentlichen auf einer sorgfältigen Anamneseerhebung, körperlichen Untersuchung und Abschätzung des aktuellen Energiebedarfs eines individuellen Patienten. SGA erlaubt es, einen individuellen Patienten ernährungsmedizinisch grob zu klassifizieren - mit den Unterscheidungen zwischen a) gut ernährt, b) mäßig mangelernährt oder c) schwer mangelernährt. Die Variablen für das SGA sind in Abbildung 1 aufgeführt. Im Besonderen wird dabei auf Gewichtsveränderungen, Veränderungen der Nahrungszufuhr, gastrointestinale Beschwerden, körperliche Aktivität und auf die Grunderkrankung mit ihrem Verhältnis zum Ernährungsbedarf geachtet. Des Weiteren werden bei der körperlichen Untersuchung der Verlust von subkutanem Fett und klinisch eindeutig erkennbarer Muskelschwund registriert. Zusätzlich festzuhalten sind Knöchel- oder Flankenödeme bzw. Aszites. 
Um zu einer entsprechenden SGA-Klassifizierung zu gelangen, wird kein im Detail festgelegter numerischer Algorithmus benutzt. Es erfolgt vielmehr eine Einstufung auf der Basis einer subjektiven Gewichtung. Trotz dieser Subjektivität findet sich eine eindeutige Korrelation des SGA mit der Prognose des chirurgischen Patienten.

\section{Abschätzung des Kalorienbedarfs}

Nach chirurgischem Trauma muss die Flüssigkeits- und Elektrolytzufuhr mit einer entsprechenden Zufuhr von Kalorien bzw. Substraten in Einklang gebracht werden. Der basale Energieumsatz kann beim Gesunden anhand von Körpergewicht (KG), Alter, Geschlecht und Körpergröße nach Harris \& Benedikt näherungsweise berechnet werden. Für den klinischen Alltag lässt sich der basale Umsatz einfach nach der Faustregel von Stein und Levine (basaler Energieumsatz $(\mathrm{kcal}) /$ pro Tag $=24 \times \mathrm{kg} \mathrm{KG})$ berechnen. In der Regel ist davon auszugehen, dass unmittelbar postoperativ nach elektiven Eingriffen der Kalorienbedarf des Patienten seinem Ruheumsatz entspricht. Wird der Patient mobilisiert, so erhöht sich diese Rate um etwa 10\%. Entwickelt der Patient ein Multiorganversagen, so sinkt der Energieumsatz um 20-30\% [7].

\section{Ernährung direkt postoperativ}

Der errechnete Kalorienbedarf entspricht nicht der empfohlenen Kalorienzufuhr direkt postoperativ: Aufgrund der Substratverwertungsstörungen im Rahmen des Postaggressionssyndroms werden am Operationstag direkt postoperativ zunächst keine Substrate zugeführt, sondern lediglich Elektrolyte und Flüssigkeit. Ab dem ersten postoperativen Tag beginnt die Energiezufuhr, die in den ersten 2-3 Tagen aufgrund des fortbestehenden Postaggressionsstoffwechsels deutlich hypokalorisch sein muss und dann im Verlauf vom 4. bis zum 7. Tag weiter bis zum berechneten Energiebedarf gesteigert wird. Eine Zufuhr von Vitaminen und Spurenelementen kann bei Patienten ohne vorbestehende Mangelzustände während der Phase der hypokalorischen Ernährung zunächst unterbleiben.

Insbesondere bei parenteraler Ernährung kann Stickstoffverlust durch Glukoneogenese begrenzt werden durch eine bedarfsgerechte Zufuhr von Aminosäuren (1,2-1,5 g AS/kg KG/Tag) ab dem ersten Tag postoperativ und eine an den Postaggressionsstoffwechsel angepasste Kalorienzufuhr.

\section{Enterale Ernährung vor parenteraler Ernährung}

Dies gilt für alle Patienten, ob operiert oder nicht operiert, nach großen und kleinen Eingriffen und auch für Intensivpatienten. Die Ernährung soll, wann immer möglich, enteral erfolgen (Grad-A-Empfehlung, DGEM-Leitlinie). Die frühe enterale Ernährung bewirkt eine Reduktion infektöser Komplikationen sowie ein rascheres Einsetzen der Peristaltik und der Darmpassage. Anzahl und Schwere postoperativer Komplikationen und Krankenhausverweildauer sind bei Patienten mit enteraler Ernährung reduziert. Kontraindikationen für eine enterale Ernährung sind:

- Funktionsuntüchtigkeit des Gastrointestinaltrakts.

- Ileus.

- Akute gastrointestinale Blutung.

- Akute diffuse Peritonitis.

- Nicht beherrschbares Erbrechen.

- Kein vorhandener Zugang zum Gastrointestinaltrakt.

- Kreislaufinstabilität/akuter Schockzustand/hochdosierter Vasopressoreneinsatz.

- Akute metabolische Entgleisung.

Enterale Ernährung kann als orale Nahrungszufuhr erfolgen oder, wenn dies nicht möglich ist, als enterale Sondenernährung.

\section{Orale Ernährung}

Die Krankenhausküche liefert Nahrung in Abhängigkeit von der angeforderten Konsistenz und vom Ballaststoffgehalt (flüssige, passierte Kost, leichte Kost, Vollkost) sowie stoffwechseladaptierte Diäten (Diabetes-Kost, kaliumarm, cholesterinfrei, glutenfrei). Zur Sicherung einer ausreichenden Kalorienzufuhr muss die tatsächlich vom Patienten verzehrte Nahrungsmenge sorgfältig dokumentiert werden. Wird die erforderliche Kalorienzufuhr nicht erreicht, sollte zusätzlich über Sondenernährung oder - falls dies nicht möglich ist parenteral ernährt werden.

\section{Enterale Sondenernährung}

Bei behindertem Schluckakt oder funktionsuntüchtigem oberem Gastrointestinaltrakt (Magen/Ösophagus) werden Substrate enteral zugeführt [8]. Bezüglich der Indikationen sowie Vor- und Nachteile der Zugangswege für die Sondenernährung wird auf die Leitlinie der DGEM verwiesen ( $w w w$. awmf-leitlinien.de).

Bei der enteralen Ernährung kommen industriell hergestellte bilanzierte Diäten zur Anwendung, die bedarfsdeckend für den Nährstoff-, Spurenelement-, Elektrolyt- und Vitaminbedarf des Patienten sind. Unterschieden werden nährstoffdefinierte Diäten (NDD) von chemisch definierten Diäten (CDD), also hochmolekulare von niedermolekularen Darreichungsformen. Die NDD sind ballaststoffarm und können bei normaler Digestions- und Resorptionsleistung gegeben werden. Sie enthalten Kohlenhydrate als Oligo- und Polysaccharide (50-60\%), intaktes Eiweiß aus Milch, Soja, Eiklar und Fleischprotein (15-20\%) und schließlich Fette aus Pflanzen- 
Tab. 1. Menge und Art der Sondenkost nach Patientengewicht und Erkrankung (Standard der Chirurgischen Intensivstation, UKSH, Campus Lübeck)

\begin{tabular}{|c|c|c|c|c|}
\hline & \multicolumn{4}{|c|}{ Patientengewicht } \\
\hline & $60 \mathrm{~kg} \mathrm{KG}$ & $70 \mathrm{~kg} \mathrm{KG}$ & $80 \mathrm{~kg} \mathrm{KG}$ & $90 \mathrm{~kg} \mathrm{KG}$ \\
\hline \multicolumn{5}{|l|}{ Sondenkost Standard } \\
\hline $\begin{array}{l}\text { Standard-Sondenkost, } 1 \mathrm{kcal} / \mathrm{ml} \text {, } \\
\text { nährstoffdefiniert, mit Ballaststoffen }\end{array}$ & $1900 \mathrm{ml} /$ Tag & $2200 \mathrm{ml} /$ Tag & $2500 \mathrm{ml} / \mathrm{Tag}$ & $2800 \mathrm{ml} / \mathrm{Tag}$ \\
\hline Gesamt-kcal/Tag & 1900 & 2200 & 2500 & 2800 \\
\hline \multicolumn{5}{|l|}{ Sondenkost Dialysepatienten } \\
\hline «Nephro»-Lösung, 2 kcal/ml, kalorienarm & $1000 \mathrm{ml} / \mathrm{Tag}$ & $1150 \mathrm{ml} / \mathrm{Tag}$ & $1300 \mathrm{ml} / \mathrm{Tag}$ & $1500 \mathrm{ml} / \mathrm{Tag}$ \\
\hline Gesamt-kcal/Tag & 2000 & 2300 & 2600 & 3000 \\
\hline \multicolumn{5}{|c|}{ Sondenkost für Kurzdarm, Maladsorptionsstörungen } \\
\hline $\begin{array}{l}\text { Oligopeptid-Diät, chemisch definiert, } \\
1 \mathrm{kcal} / \mathrm{ml} \text {, ballaststofffrei }\end{array}$ & $1800 \mathrm{ml} / \mathrm{Tag}$ & $2100 \mathrm{ml} / \mathrm{Tag}$ & $2400 \mathrm{ml} / \mathrm{Tag}$ & $2700 \mathrm{ml} / \mathrm{Tag}$ \\
\hline Gesamtkalorien & 1800 & 2100 & 2400 & 2700 \\
\hline \multicolumn{5}{|c|}{$\begin{array}{l}\text { Zusätzlich zu den aufgeführten Sondenformulierungen kann eiweißreiche Trinknahrung verordnet werden }(2 \times 200 \mathrm{ml} / \mathrm{Tag} \\
\text { mit entweder } 10 \mathrm{~g} \text { Eiweiß/100 ml oder mit 5,6 g Eiweiß/100 ml und } 100 \mathrm{bzw} .150 \mathrm{kcal} / 100 \mathrm{ml}) \text {, die getrunken oder auch per } \\
\text { Sonde gegeben werden kann. } \\
\text { Es wird kein Wasser zur Sondenkost zugemischt. Wasser wird jeweils zu den Medikamentengaben gegeben. Dafür wird die } \\
\text { Sondenkost pausiert (Vermeidung von Interaktionen). Standardverordnung: } 2 \times 250 \mathrm{ml} \text { Wasser/Tag. }\end{array}$} \\
\hline
\end{tabular}

ölen (25-30\%), wobei die Zufuhr von essenziellen Fettsäuren gesichert ist. Für katabole Patienten sind Diäten mit höherer Kaloriendichte und erhöhtem Eiweißgehalt zur eiweißreichen Ernährung (1,3-1,5 g/kg KG/Tag) verfügbar.

Für eine unkomplizierte Langzeiternährung bei uneingeschränktem, funktionstüchtigem Gastrointestinaltrakt existieren auch ballaststoffhaltige Nahrungen. Hier müssen Diäten mit unlöslichen Ballaststoffen (Zellulose) von solchen mit löslichen Ballaststoffen (Präbiotika) unterschieden werden. $\mathrm{Zu}$ letzteren zählen Pektine, Agar, Pflanzenschleim und Oligofruktosen.

Die CDD kommen bei globalen Störungen der intraluminalen Hydrolysekapazität und Resorption zum Einsatz, z.B. bei ausschließlich jejunaler Ernährung postoperativ unter Umgehung der Magenpassage, bei Patienten mit Kurzdarmsyndrom, aber eventuell auch bei septischen Patienten oder Patienten mit gastrointestinalen Resorptionsstörungen. CDD sind arm an Fett, ballaststofffrei und gering hyperosmolar, d.h. sie enthalten Aminosäuren, Tripeptide oder Oligopeptide. Diese Oligopeptiddiäten werden nahezu vollständig im oberen Gastrointestinaltrakt resorbiert. Ihr genereller Einsatz als Standardtherapie bei kritisch Kranken ist jedoch nicht angezeigt.

\section{Stoffwechseladaptierte Diäten}

Sogenannte stoffwechseladaptierte Diäten sind für Patienten mit spezifischen Organerkrankungen und Insuffizienzen sowie für Situationen mit metabolischen Besonderheiten entwickelt worden. Zu diesen speziellen Diäten zählen die Diabetesdiäten, Diäten mit immunmodulatorischer Wirkung (Arginin, Glutamin, Omega-3-Fettsäuren, Dihomogamma-Linolensäure, Antioxidantien), leberadaptierte Sondendiäten und schließlich nierenadaptierte Diäten (kalorienarm, flüssigkeits- reduziert, höhere Energiedichte). Spezielle Arginin-freie Diäten sind eventuell bei beatmeten Patienten von klinischem Nutzen. Für Arginin-haltige Diäten ist jedoch speziell bei Intensivpatienten weder eine Verbesserung der Morbidität noch der Letalität nachgewiesen, sodass der Einsatz dieser speziellen Produkte bei kritisch kranken Patienten gegenwärtig nicht empfohlen wird. Bei mangelernährten onkologischen Patienten, die postoperativ kein Organversagen aufweisen, besteht eine klare Empfehlung zur prä- und postoperativen Immunonutrition einschließlich der Arginin-Applikation. Die DGEM-S3-Leitlinie zur Ernährung von Intensivpatienten (gültig bis 9/2011) empfiehlt immunmodulierende Diäten für Patienten mit Operationen am oberen Gastrointestinaltrakt (Ösophagus, Magen, Pankreas) (Grad A) und Traumapatienten. Ausdrücklich nicht empfohlen sind immunmodulierende Diäten für Patienten mit schwerer Sepsis [9]. Patienten mit akutem Atemnotsyndrom (ARDS) können von Formulierungen mit Omega-3-Fettsäuren und Antioxidantien profitieren (Empfehlung Grad B).

\section{Praktische Umsetzung der Empfehlungen zur Sonden- ernährung (Standard der chirurgischen Intensivstation am Universitätsklinikum Schleswig-Holstein, Campus Lübeck)}

Im klinischen Alltag können schriftlich festgelegte Standards für die Sondenernährung die Anwendung einer leitliniengerechten und patientenadaptierten Ernährung erleichtern. In Tabelle 1 finden sich als Beispiel für einen solchen Standard die an das Patientenkörpergewicht (Sollgewicht) adaptierten Mengen an Sondennahrung aufgeführt und welche Sondenkost für welche Patienten verwendet werden soll. Für Patienten mit großer onkologischer Viszeralchirurgie am obe- 
ren Gastrointestinaltrakt und für Polytraumapatienten steht eine sog. Immunonutritionslösung zur Verfügung, die $1 \mathrm{kcal} / \mathrm{ml}$ enthält und die als Standardsondenkost dosiert wird. Eine Diabetiker-Sondenkost wird am Universitätsklinikum Lübeck nicht vorgehalten. Die Kalorien/BE können auch mit den vorhandenen Sondenformulierungen umgesetzt werden.

Die Sondennahrung wird mittels Ernährungspumpen gegeben und läuft kontinuierlich ohne nächtliche Pause. Die Zufuhr erfolgt in der Regel über eine - meist endoskopisch gelegte - nasojejunale 3-Lumen-Ernährungssonde, die bei Ernährung über den jejunalen Schenkel der Sonde einen Ablauf von Magensekret bei Magenatonie erlaubt. Eine solche Sonde wird auch intraoperativ eingelegt und distal der Anastomosen bei Chirurgie am oberen Gastrointestinaltrakt zur frühen enteralen Ernährung platziert. Bei Patienten mit intakten Reflexen und ohne gastralen Reflux ist auch eine gastrale Ernährung möglich.

Erforderliche zusätzliche Flüssigkeitssubstitution wird in Form von Wasser verordnet. Vor und nach Medikamentengabe wird die Sonde mit Wasser gespült (nach jedem Medikament!).

Bei Medikamenten, die nicht in flüssiger Form angeordnet sind, ist darauf zu achten, ob die Auflösung oder Zermörserung des Medikaments die Wirkung nicht beeinträchtigt und ob das Medikament jejunal oder gastral appliziert werden muss. Bei Fragen ist die Klinikapotheke oder die Internet-

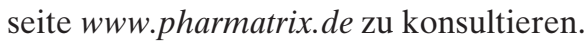

\section{Parenterale Ernährung}

Bei funktionsuntüchtigem unterem Gastrointestinaltrakt (Dünndarm/Dickdarm) muss die Ernährung parenteral erfolgen. Sie ist in der ersten Woche nach chirurgischem Trauma hypokalorisch, ab der zweiten Woche normo(iso-)kalorisch. Sowohl für die frühe (Tag 1-3) als auch für die späte (Tag 4-6) postoperative Phase stehen kommerziell erhältliche Infusionslösungen zur Verfügung, die dem jeweiligen Flüssigkeitsbedarf in diesem Zeitraum (40 ml/kg KG/Tag in der frühen und $25-30 \mathrm{ml} / \mathrm{kg} \mathrm{KG/Tag} \mathrm{in} \mathrm{der} \mathrm{späten} \mathrm{postoperativen} \mathrm{Phase)}$ Rechnung tragen. Die Infusionslösungen für die frühe postoperative Phase sind leicht hyperton (700-800 mosm/l) und besitzen eine geringe kalorische Dichte $(0,3-0,4 \mathrm{kcal} / \mathrm{ml})$. Sie enthalten 5\% Zucker, 3,5\% Aminosäuren und Elektrolyte entsprechend den Erhaltungsdosen. Die Konzentrationen sind so angelegt, dass bei Zufuhr von $40 \mathrm{ml} / \mathrm{kg} /$ Tag eine Substratzufuhr erfolgt, die den in der frühen postoperativen Phase bestehenden Substratverwertungsmöglichkeiten entspricht. Die niedrige Osmolarität dieser Lösungen erlaubt die Zufuhr über einen peripher venösen Zugang.

$\mathrm{Ab}$ dem 4. postoperativen Tag wird dann die zugeführte Kalorien- bzw. Kohlenhydratmenge erhöht. Die kommerziell verfügbaren Lösungen, die in dieser Situation zum Einsatz kommen, enthalten hypertone Kohlenhydrate, Aminosäuren, Spurenelemente und Elektrolyte. Dem geringeren Flüssig- keitsbedarf in dieser Phase der Erkrankung entspricht die höhere Kaloriendichte, sodass diese Lösungen mit 25-30 ml/kg $\mathrm{KG} /$ Tag infundiert werden können. Wegen der hohen Osmolarität (>1300 mosm/l) müssen diese Lösungen über einen zentral venösen Katheter zugeführt werden. Die kommerziell verfügbaren Infusionslösungen sind so zusammengesetzt, dass sie dem gesteigerten Eiweißbedarf (1,2-1,5 g/kg/Tag) des Patienten Rechnung tragen. Im Gegensatz zur frühen postoperativen Ernährung tritt jedoch jetzt ein erhöhter Kohlenhydratanteil hinzu. Die Anwendung der Kompletternährungslösungen bedeutet für die Pflegekräfte einen geringeren Aufwand, und es werden weniger Infusionspumpen benötigt.

Bei chirurgischen Intensivpatienten sind Hyperchlorämie und Hypernatriämie häufig, insbesondere nach massiver Volumensubstitution, da alle kommerziell erhältlichen Lösungen mit diesen Elektrolyten «belastet» sind. Zudem haben kritisch kranke Patienten Störungen des Glukosestoffwechsels, die eine Variabilität der Glukosezufuhr unabhängig von der Aminosäurenzufuhr erfordern. Hinzu kommen möglicherweise andere Wasser- und Elektrolytunausgewogenheiten, denen Komplett-Ernährungslösungen nicht gerecht werden.

In diesen Fällen sind Einzellösungen von Aminosäuren und Zucker nach den zuvor aufgezeigten Prinzipien miteinander zu kombinieren (Komponententrennung). Aminosäurelösungen sind mit und ohne Elektrolytzusatz im Handel erhältlich. Sie sind in der Regel 10\%ig und enthalten synthetische kristalline Aminosäuren, und zwar meist zu 40-50\% essenzielle Aminosäuren; der Rest sind nicht essenzielle Aminosäuren.

\section{Glutamin}

Bei längerfristiger total parenteraler Ernährung oder bei chronisch inadäquater enteraler Ernährung muss zusätzlich Glutamin in Form von Glutamin-haltigen Dipeptiden zugeführt werden [10]. Die empfohlene Tagesdosis beträgt 0,3-0,4 g Glutamin/kg KG/Tag. Die Tageshöchstdosis beträgt $2 \mathrm{ml} / \mathrm{kg}$ KG/Tag. Tabelle 2 zeigt den Standard der chirurgischen Intensivstation des Universitätsklinikums Schleswig-Holstein (UKSH), Campus Lübeck.

Beim gesunden Erwachsenen liegt die notwendige Eiweißmenge bei etwa $0,8 \mathrm{~g} / \mathrm{kg} \mathrm{KG} / \mathrm{Tag}$. Intensivpatienten nach komplikationslosen größeren chirurgischen Eingriffen benötigen je nach Ausmaß ihrer Erkrankung zwischen 1,2 und 1,5 g Eiweiß/kg KG/Tag und damit mehr Eiweiß als ein nicht intensivpflichtiger Patient nach einer vergleichbaren Operation.

Die Konzentrationen für Zuckerlösungen bewegen sich zwischen 10 und 40\%. Die derartig applizierten Kohlenhydratmengen müssen selbstverständlich dem Schweregrad des Postaggressionsstoffwechsels angepasst und dementsprechend dosiert werden [11].

Zur kompletten isokalorischen parenteralen Ernährung (frühestens in der zweiten postoperativen Woche) existieren hyperosmolare Aminosäure/Kohlenhydrat/Fettkombinationslösungen. Fett kann jedoch auch als Einzelbestandteil der parenteralen Ernährung getrennt zugeführt werden. 
Tab. 2. Standard für den Einsatz von Glutamin in der TPE bei Intensivpatienten (Chirurgische Intensivstation des UKSH, Campus Lübeck)

\begin{tabular}{ll}
\hline Präparat & $\begin{array}{l}\text { Glutaminlösung als Zusatz zu Aminosäurelösungen (Alternative: Komplett- } \\
\text { Aminosäurelösung mit Glutamin) }\end{array}$ \\
\hline Indikation & $\begin{array}{l}\text { Intensivpatienten mit TPE: insbesondere auch Patienten mit Sepsis, } \\
\text { Polytrauma, Verbrennung mit TPE, Patienten nach großen operativen } \\
\text { Eingriffen (Gastrektomie/Ösophagusresektion) mit TPE }\end{array}$ \\
\hline Kontraindikation & $\begin{array}{l}\text { Kreatinin-Clearance von }<25 \mathrm{ml} / \text { min } \\
\text { Leberinsuffizienz ab Child B } \\
\text { Patienten mit partiell enteraler Ernährung, wenn TPE reduziert wurde }\end{array}$ \\
& Patienten mit schwerer nekrotisierender akuter Pankreatitis \\
\hline Patienten mit CVVHDF und SLEDD & bekommen Dipeptamin, wenn sie täglich behandelt werden, da dann eine \\
Clearance von mindestens 35 ml/min erreicht wird
\end{tabular}

\section{Auf Organfunktionsstörungen zugeschnittene}

\section{Aminosäurelösungen}

Vergleichbar mit der enteralen Ernährung existieren auch für die parenterale Ernährung spezifische Präparate zur Kompensation eventueller Organfunktionsstörungen [12, 13]. Diese sog. Nierenlösungen enthalten ausschließlich essenzielle und wenige semiessenzielle Aminosäuren. Sie können bei Patienten mit Nierenversagen angewandt werden. Belastbare Daten, die einen Benefit des Patienten belegen, gibt es allerdings nicht.

Für Patienten mit hepatischer Enzephalopathie und einer Ammoniakkonzentration von mehr als $100 \mathrm{mg} / \mathrm{dl}$ existieren ebenfalls parenteral einsetzbare Leberlösungen mit einer erhöhten Zufuhr an verzweigtkettigen Aminosäuren.

Eine Applikation von Vitaminen und Spurenelementen ist postoperativ nur bei lang dauernder parenteraler Ernährung (länger als eine Woche) erforderlich. Es existieren kommerzielle Präparate, die die wichtigsten Spurenelemente (Chrom, Kupfer, Eisen, Mangan, Fluor, Molybdän, Selen und Zink) enthalten und die in der Regel täglich zugeführt werden. Wasserlösliche und fettlösliche Vitamine müssen bei längerer parenteraler Ernährung substituiert werden. Es gibt Komplettpräparate, die den Tagesbedarf eines Erwachsenen abdecken. Zusätzlich können besonders wichtige Spurenelemente (Selen) einzeln parenteral appliziert werden [14].

Als Beispiel für eine leitliniengerechte Umsetzung parenteraler Ernährung bei Intensivpatienten zeigt Tabelle 3 den diesbezüglichen Standard der chirurgischen Intensivstation des UKSH, Campus Lübeck. In dem Standard wurde bewusst auf Komplettlösungen verzichtet, um variabler auf Elektrolyt-, Blutzucker- und andere Stoffwechselunausgewogenheiten eingehen zu können. Der Blutzuckerspiegel der Patienten mit totaler parenteraler Ernährung (TPE) wird auf einen Zielwert von $150 \mathrm{mg} \%$ eingestellt. Werden hierfür beim Nicht-Diabetiker über längere Zeit Insulinmengen von mehr als 8 IE Insulin/h benötigt, wird die zugeführte Zuckermenge reduziert. Im Standard angegebene gewichtsbezogene Dosierungen erleichtern die ärztlichen Verordnungen.

\section{Spezielle Aspekte der Ernährung nach großen viszeralchirurgischen Eingriffen}

Auch bei chirurgischen Patienten, die im Rahmen ihres chirurgischen Eingriffs Anastomosen am Gastrointestinaltrakt zur Wiederherstellung der Kontinuität erhalten haben, ist generell postoperativ eine Unterbrechung der oralen/enteralen Nahrungszufuhr nicht erforderlich. Der orale/enterale Kostaufbau sollte sich vor allem nach der Toleranz des Patienten richten. Nach Anastomosen am Kolon und Rektum kann ab dem 1. postoperativen Tag mit der oralen/ enteralen Nahrungszufuhr begonnen werden. Bei Anastomosen am oberen Gastrointestinaltrakt ist für die ersten 
Tab. 3. Standardinfusionsprogramme für die TPE; Chirurgische Intensivstation des UKSH Campus Lübeck. Der Standard erhebt keinen Anspruch auf Allgemeingültigkeit. In diesem Standardplan wird nur eine parenterale Ernährung mit Einzelkomponenten abgebildet. Denkbar sind auch ähnliche Vorgehensweisen mit Komplettlösungen

\begin{tabular}{|c|c|c|c|c|}
\hline & \multicolumn{4}{|c|}{ Patientengewicht } \\
\hline & $60 \mathrm{~kg} \mathrm{KG}$ & $70 \mathrm{~kg} \mathrm{KG}$ & $80 \mathrm{~kg} \mathrm{KG}$ & $90 \mathrm{~kg} \mathrm{KG}$ \\
\hline \multicolumn{5}{|c|}{ Hypokalorische Ernährung in der Postaggressionsphase (Tag 1-3 postoperativ) } \\
\hline $\begin{array}{l}\text { Komplettlösung niedrigkalorisch (AS } 35 \mathrm{~g} / 1 \text {, } \\
\text { Gluc } 50 \mathrm{~g} / 1 \text {, mit Elektrolyten) }\end{array}$ & $1750 \mathrm{ml} / \mathrm{Tag}$ & $2000 \mathrm{ml} /$ Tag & $2250 \mathrm{ml} / \mathrm{Tag}$ & $2500 \mathrm{ml} / \mathrm{Tag}$ \\
\hline Gesamt-kcal & 595 & 680 & 765 & 850 \\
\hline \multicolumn{5}{|c|}{ Vollkalorische parenterale Ernährung (ab Tag 4 postoperativ anstreben) ${ }^{a}$} \\
\hline $\begin{array}{l}\text { 10\%ige Komplett-Aminosäurelösung } \\
\text { mit Elektrolyten }\end{array}$ & $900 \mathrm{ml} / \mathrm{Tag}$ & $1050 \mathrm{ml} / \mathrm{Tag}$ & $1200 \mathrm{ml} / \mathrm{Tag}$ & $1350 \mathrm{ml} / \mathrm{Tag}$ \\
\hline Glukose $40 \%$ & $600 \mathrm{ml} / \mathrm{Tag}$ & $700 \mathrm{ml} / \mathrm{Tag}$ & $800 \mathrm{ml} / \mathrm{Tag}$ & $900 \mathrm{ml} / \mathrm{Tag}$ \\
\hline Fettlösung auf Olivenölbasis $20 \%$ ig & $350 \mathrm{ml} / \mathrm{Tag}$ & $450 \mathrm{ml} / \mathrm{Tag}$ & $500 \mathrm{ml} /$ Tag & $600 \mathrm{ml} / \mathrm{Tag}$ \\
\hline Gesamt-kcal & 2020 & 2440 & 2760 & 3180 \\
\hline
\end{tabular}

Kompensierte chronische Niereninsuffizienz ohne Dialyse (kein akutes Nierenversagen, keine kontinuierliche Nierenersatztherapie und keine schwere Katabolie, in letzterem Fall mehr AS erforderlich) ${ }^{a}$

\begin{tabular}{|c|c|c|c|c|}
\hline $\begin{array}{l}\text { 10\%ige Komplett-Aminosäurelösung } \\
\text { ohne Elektrolyte }\end{array}$ & $750 \mathrm{ml} / \mathrm{Tag}$ & $900 \mathrm{ml} / \mathrm{Tag}$ & $1000 \mathrm{ml} / \mathrm{Tag}$ & $1150 \mathrm{ml} / \mathrm{Tag}$ \\
\hline Glukose $40 \%$ & $600 \mathrm{ml} / \mathrm{Tag}$ & $700 \mathrm{ml} / \mathrm{Tag}$ & $800 \mathrm{ml} / \mathrm{Tag}$ & $900 \mathrm{ml} / \mathrm{Tag}$ \\
\hline Fettlösung auf Olivenölbasis $20 \%$ ig & $350 \mathrm{ml} / \mathrm{Tag}$ & $450 \mathrm{ml} / \mathrm{Tag}$ & $500 \mathrm{ml} / \mathrm{Tag}$ & $600 \mathrm{ml} / \mathrm{Tag}$ \\
\hline Gesamt-kcal & 1960 & 2380 & 2680 & 3100 \\
\hline \multicolumn{5}{|l|}{ Dialyse bei chronischem Nierenversagen ${ }^{b}$} \\
\hline $\begin{array}{l}10 \% \text { ige Komplett-Aminosäurelösung } \\
\text { ohne Elektrolyte }\end{array}$ & $900 \mathrm{ml} / \mathrm{Tag}$ & $1050 \mathrm{ml} /$ Tag & $1200 \mathrm{ml} / \mathrm{Tag}$ & $1350 \mathrm{ml} /$ Tag \\
\hline Glukose $40 \%$ & $600 \mathrm{ml} / \mathrm{Tag}$ & $700 \mathrm{ml} / \mathrm{Tag}$ & $800 \mathrm{ml} / \mathrm{Tag}$ & $900 \mathrm{ml} / \mathrm{Tag}$ \\
\hline Fettlösung auf Olivenölbasis $20 \%$ ig & $350 \mathrm{ml} / \mathrm{Tag}$ & $450 \mathrm{ml} / \mathrm{Tag}$ & $500 \mathrm{ml} / \mathrm{Tag}$ & $600 \mathrm{ml} / \mathrm{Tag}$ \\
\hline Gesamt-kcal & 2020 & 2440 & 2760 & 3180 \\
\hline
\end{tabular}

Kontinuierliche Nierenersatztherapie (CVVHDF oder SLEDD) bei akutem oder chronischem Nierenversagen ${ }^{b}$

\begin{tabular}{|c|c|c|c|c|}
\hline $\begin{array}{l}\text { 10\%ige Komplett-Aminosäurelösung } \\
\text { mit Elektrolyten }\end{array}$ & $1000 \mathrm{ml} / \mathrm{Tag}$ & $1150 \mathrm{ml} / \mathrm{Tag}$ & $1300 \mathrm{ml} / \mathrm{Tag}$ & $1500 \mathrm{ml} / \mathrm{Tag}$ \\
\hline Glukose $40 \%$ & $600 \mathrm{ml} / \mathrm{Tag}$ & $700 \mathrm{ml} / \mathrm{Tag}$ & $800 \mathrm{ml} / \mathrm{Tag}$ & $900 \mathrm{ml} / \mathrm{Tag}$ \\
\hline Fettlösung auf Olivenölbasis $20 \%$ ig & $350 \mathrm{ml} / \mathrm{Tag}$ & $450 \mathrm{ml} / \mathrm{Tag}$ & $500 \mathrm{ml} / \mathrm{Tag}$ & $600 \mathrm{ml} / \mathrm{Tag}$ \\
\hline Gesamt-kcal & 2060 & 2480 & 2800 & 3240 \\
\hline
\end{tabular}

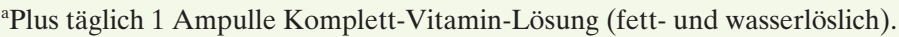

bPlus täglich 1 Ampulle Vitamin-Lösung wasserlösliche und 3-mal pro Woche 1 Ampulle fettlösliche Vitamine. Werden über längere Zeit Insulinmengen von mehr als 8 IE Insulin/h benötigt, um einen Blutzucker von $150 \mathrm{mg} \% \mathrm{zu}$ erreichen, wird die zugeführte Glukosemenge reduziert. Fettlösliche Vitamine werden zusammen mit der Fettlösung gegeben. Ab Tag 4 bekommen alle Patienten eine Ampulle Spurenelemente täglich.

Patienten mit akutem und mit chronischem Nierenversagen bekommen zusätzlich 3-mal pro Woche 0,5 g Vitamin C. Patienten mit akutem Nierenversagen bekommen zudem 1-mal täglich $1 \mu \mathrm{g}$ EinsAlpha (jedoch nicht, wenn SeCalcium $>2,6 \mathrm{mmol} / \mathrm{l})$.

Tage die enterale Nahrungszufuhr über eine distal der Anastomose liegenden Sonde/Feinnadelkatheterjejunostomie zu empfehlen.

Bei abdominalchirurgischen Eingriffen ohne Verletzungen der Integrität des Intestinaltrakts kann ebenfalls in Abhängigkeit vom Ausmaß des Traumas bereits am 1. postoperativen Tag mit dem oralen Kostaufbau begonnen werden. Nach kurzfristiger Verabreichung von überwiegend flüssiger Kost kann dabei bereits ab dem 2. postoperativen Tag auf die Verabreichung von ballaststoffarmer leichter Kost übergegangen werden. Auch hier ist jedoch die tatsächlich verzehrte Kalorienmenge zu kontrollieren. Dabei werden die zuzuführenden Kalorienmengen - wie bei der parenteralen Ernährung - dem Verlauf des Postaggressionssyndroms angepasst.
Bei Eingriffen am unteren Gastrointestinaltrakt erfolgt der orale Kostaufbau leicht verzögert, mit zunächst Zufuhr von flüssiger Nahrung in den ersten 2 Tagen, und dann mit sukzessivem Kostaufbau. Bei Eingriffen am oberen Gastrointestinaltrakt, insbesondere bei Resektionen am Magen und Ösophagus, erfolgt die Nahrungszufuhr über eine intraoperativ eingebrachte spezielle Ernährungssonde oder über eine Katheterjejunostomie. Am 1. postoperativen Tag wird mit einer kontinuierlichen Gabe Sondenkost von etwa $250 \mathrm{ml}$ über $24 \mathrm{~h}$ begonnen. Die tägliche Sondenmenge wird dann in den nächsten Tagen, sofern keine ausreichende orale Nahrungszufuhr erfolgen kann, kontinuierlich um $250 \mathrm{ml} / 24 \mathrm{~h}$ gesteigert, bis das kalorische Maximum von $24 \mathrm{kcal} / \mathrm{kg} \mathrm{KG}$ erreicht ist. 
Bei gastraler, aber auch bei jejunaler Ernährung über eine Sonde muss auf Passagestörungen geachtet werden. Bei insuffizienter enteraler Kalorienzufuhr ist additiv eine parenterale Supplementierung durchzuführen.

Es gilt heute als gesichert, dass von einer postoperativen enteralen Sondenernährung vor allem Patienten nach großen viszeralchirurgischen Tumoroperationen oder schwerem Polytrauma profitieren. Sollte eine enterale Ernährung längerfristig ( $>4$ Wochen) erforderlich sein, so empfiehlt sich der Umstieg auf eine transkutane Sonde, z.B. als perkutane endoskopische Gastrostomie (PEG). Bei onkologischen Patienten tritt eine Besonderheit hinzu. Hier ist die postoperative Fortführung der präoperativen immunmodulierenden Sondenernährung empfohlen. Bei unkompliziertem Verlauf ist dabei ein Zeitraum von 5-7 Tagen postoperativ ausreichend.

\section{Praxis der enteralen Ernährung}

Zur enteralen Ernährung wird ein Zugang zum Gastrointestinaltrakt benötigt; dabei sind transnasale Sonden am einfachsten zu platzieren. Ist über einen absehbaren Zeitraum der obere Gastrointestinaltrakt nicht benutzbar, so sollte bereits intraoperativ eine Feinnadelkatheterjejunostomie oder eine nasojejunale 3-Lumen-Sonde angelegt werden. Zur nasogastralen Ernährung können großlumige Sonden (12-14 Charriere) verwendet werden, die gleichzeitig eine Dekompression des Magens erlauben [15].

Zwei verschiedene Applikationsmethoden - kontinuierliche Zufuhr oder Bolusgabe mit Portionen von 50-300 ml (mit oder ohne Gabe von Prokinetika) - sind grundsätzlich möglich. Allgemein zeigt die kontinuierliche Applikation eine bessere Toleranz mit höherer Energie- und Substratzufuhr, da hierunter die Raten an therapielimitierender Diarrhö und Aspirationsereignissen geringer sind. Andererseits können plötzlich auftretende Passagestörungen leicht übersehen werden, woraus sich in der Folge lebensbedrohliche Aspirationsereignisse entwickeln können. Bei unklarer Funktionslage sollte somit die manuelle Bolusapplikation zur Anwendung kommen, da das Pflegepersonal vor jeder erneuten Instillation das im Magen vorhandene Residualvolumen überprüfen und gegebenenfalls die zu applizierende Nahrungsmenge daraufhin reduzieren kann [8].

Ferner ist zu berücksichtigen, dass eine Reihe von Faktoren die normale gastrale Motilität beeinträchtigen kann (sog. Oberbauchatonie). In hartnäckigen Fällen können hier motilitätssteigernde Pharmaka, wie Metoclopramid oder Erythromyzin (Off-Label-Use), zum Einsatz kommen. Bei einer endoskopisch nasojejunal platzierten 3-Lumen-Sonde ist ein gastraler Reflux über das proximale Lumen kein Hindernis für eine Sondenernährung.

\section{Komplikationen der enteralen Ernährung}

Beobachtet werden können gastrointestinale (Übelkeit, Diarrhö, Distension) und infektiöse Probleme (Aspirationspneumonie). Wiederholtes Auftreten von großen Magenresidual- volumina oder anhaltender Reflux über den gastralen Schenkel einer jejunalen Ernährungssonde sind ein Indikator einer funktionellen Magenentleerungsstörung.

Ein weiterer Nebeneffekt der enteralen Ernährung besteht in einer Anhebung des gastralen pH. Wegen dieses Nebeneffekts der enteralen Ernährung benötigen nur Risikopatienten (wie z.B. bei benannter gastroösophagealer Refluxerkrankung, vorhandenem Ulcus ventriculi et duodeni, HP (Helicobacter pylori)-Eradikation, katecholaminpflichtige instabile kritisch Kranke) eine Stressulkusprophylaxe mit Antazida, jedoch keinesfalls alle operierten viszeralchirurgischen Patienten; nicht einmal alle Intensivpatienten.

Die Anhebung des gastralen $\mathrm{pH}$ durch enterale Ernährung bei gleichzeitig verabreichten Antazida kann aber auch einen negativen Effekt haben: nämlich Keimaszension und pathologische Keimbesiedlung im Ösophagus und Pharynx. Treten klinisch oft nicht fassbare Mikroaspirationen unter solchen Bedingungen auf, nimmt das Risiko einer nosokomialen Pneumonie deutlich zu. Dieser Pathomechanismus kann dadurch abgeschwächt werden, dass bei gastraler Ernährung eine nächtliche Ernährungspause eingelegt wird.

Die häufigste Komplikation der enteralen Ernährung ist die Diarrhö. Die Ursachen der Diarrhö sind multifaktoriell. Die Zusammensetzung der gewählten Nährlösung (Hyperosmolarität) ist jedoch nur selten Ursache des Problems. Entscheidende Pathomechanismen sind dabei infektiöse Erkrankungen des Intestinaltrakts oder Motilitäts-, Resorptions- und Durchblutungsstörungen. Einen Sonderfall stellt die Antibiotika-induzierte pseudomembranöse Kolitis dar, die einer besonderen Diagnostik (Nachweis von Clostridium-Antitoxin im Stuhl) und einer besonderen Therapie (Vancomyzin oder Metronidazol oral/enteral) bedarf.

Ansonsten kann die Behandlung durch Reduktion der Volumenflussrate, durch den Wechsel des Ernährungsregimes bzw. der Ernährungsmodalität oder durch Umsetzen einer Antibiotikatherapie erfolgen. In schweren Fällen (hämorrhagische Kolitis) kann sogar eine komplette Ernährungspause angezeigt sein.

Neben Motilitätsstörungen im Oberbauch spielen auch Passagestörungen im Dünn- und Dickdarm bzw. im Dickdarm isoliert (Ogilvie-Syndrom/Pseudoobstruktion des Kolons) eine Rolle. Der kombinierte Dünn-/Dickdarmileus kann vom isolierten paralytischen Dickdarmileus in der Regel mittels einer Röntgenaufnahme des Abdomens differenziert werden. Bei der Pseudoobstruktion handelt es sich um eine isolierte Überblähung des Kolons mit maximaler Distension der Zökalregion.

Die Ursachen für diese Paralyse sind vielfältig; neben lokalen sowie globalen Durchblutungsminderungen (schlechtes Herzzeitvolumen) kommen entzündliche Prozesse im Abdomen und am Darm selbst sowie auch motilitätshemmende Medikamente infrage. Die Therapie erfolgt in der Regel über mechanische Maßnahmen (Hebe-Senk-Einlauf) und durch Prokinetika (Ceruletid, Prostigmin), die zum Teil mehrmals 
täglich gegeben werden müssen, um entsprechende Fortschritte in der Passage zu erzielen. Bei einer Kolonüberblähung von mehr als $10 \mathrm{~cm}$ Durchmesser ist eine endoskopische Dekompression angezeigt, z.B. durch Luftabsaugung oder Legen einer Darmdekompressionssonde. In Extremfällen kann es durch die Überdehnung zur Darmwandischämie, Perforation oder Peritonitis kommen und somit eine Operation erforderlich machen.

\section{Besonderheiten in der Ernährung bei SIRS/septischen Patienten}

Auch bei septischen Patienten oder bei einem SIRS sollte frühzeitig mit einer enteralen Ernährungstherapie begonnen werden. Letztere ist einer hypokalorischen oralen Nahrungszufuhr oder einer erst später begonnenen enteralen Ernährung überlegen. In akuten Schockzuständen bzw. bei schwerer Kreislaufinsuffizienz sollte auch bei diesen Patienten auf jegliche Form der Ernährungstherapie komplett verzichtet werden.

Im Mittelpunkt der ernährungsmedizinischen Anstrengungen bei chronisch septischen Patienten steht die Bekämpfung der Eiweißkatabolie. So werden bei Patienten mit schwerem SIRS oder schwerer Sepsis im Zeitraum von etwa 3 Wochen zwischen 1,2 und 1,4 kg reines Eiweiß verloren; das entspricht etwa 13\% des Ausgangswertes. Die Masse des Eiweißverlustes entfällt dabei auf die Skelettmuskulatur. Die Höhe der Kalorienzufuhr in Relation zum Gesamtenergieumsatz hat dabei keinen wesentlichen Einfluss auf die Eiweißkatabolie. Auch bei hoher Eiweißzufuhr wird die Eiweißkatabolie zwar reduziert, jedoch nicht gänzlich aufgehoben. Es besteht ein Optimum für die tägliche Eiweißzufuhr, das etwa 1,5 g Eiweiß/kg KG/Tag beträgt. Darüber hinaus sind keine günstigen Effekte auf den Eiweißstoffwechsel zu erwarten. Allerdings kann die enterale Ernährung im Vergleich zu einer isokalorischen parenteralen Ernährung den Eiweißverlust des Intensivpatienten über einen Zeitraum von 10 Tagen fast halbieren.

Spezielle Empfehlungen existieren für Patienten unter mechanischer Nierenersatztherapie bzw. bei ausgeprägter Adipositas. Speziell unter kontinuierlicher Hämofiltration besteht ein deutlich erhöhter Eiweißbedarf (bis zu 2,5 g/kg/Tag). Ähnlich hohe Zufuhrraten werden auch für adipöse Patienten empfohlen (2,0 g/kg Idealgewicht/Tag für BMI 30-40; 2,5 g/kg Idealgewicht/Tag für BMI > 40).

\section{Besonderheiten beim Kalorienbedarf}

Im Gegensatz zu elektiven chirurgischen Operationen kann der gesamte Energieumsatz als Summe des Ruheenergieumsatzes und des Aktivitätsenergieumsatzes bei schwerer Sepsis bzw. SIRS von etwa $2000 \mathrm{kcal}$ in der 1 . Woche auf bis zu $4000 \mathrm{kcal}$ in der 2. Woche nach chirurgischer Homöostasestörung ansteigen. Somit weisen diese Patienten in der Akutphase ihres Krankheitsgeschehens eine zusätzliche Steigerung um 40-50\% über den Ruheenergieumsatz hinaus auf. Bemer- kenswert ist jedoch, dass bei der Entwicklung eines septischen Mehrfachorganversagens wieder eine Abnahme des Energiebedarfs zu beobachten ist (in der Regel nur mehr 10-20\% über dem Ruheenergieumsatz).

Gerade bei septischen Patienten ist jedoch eine Steuerung der Kalorienzufuhr nach tatsächlichem Energieumsatz nicht angezeigt. Die Begründung ist darin zu suchen, dass auch eine sehr hohe Kalorienzufuhr die Katabolie der endogenen Substratspeicher nicht aufhalten kann und sich somit nicht metabolisch günstig auswirkt. Bereits eine Erhöhung der Kalorienzufuhr um 20\% über den Ruheenergieumsatz hinaus führt bei Intensivpatienten so nur zu einer Zunahme der Fettmasse. Somit gilt für die überwiegende Mehrheit der septischen Patienten, dass eine konservative Kalorienzufuhr zwischen 21-25 $\mathrm{kcal} / \mathrm{kg} \mathrm{KG/Tag} \mathrm{ausreicht.} \mathrm{Eine} \mathrm{Kalorienzufuhr} \mathrm{in} \mathrm{derartiger}$ Höhe stellt einen Kompromiss dar zwischen dem Versuch, körpereigene Verluste soweit wie möglich zu kompensieren, und dem Ziel, toxische Nebeneffekte zu vermeiden.

Für bestimmte Intensivpatienten scheint zu gelten, dass nach Adjustierung an potenzielle Störgrößen (Alter, Aufnahmediagnose, Apache-II-Score bei Aufnahme) - im Bereich zwischen 500 bis $2000 \mathrm{kcal}$ eine signifikante inverse Beziehung zwischen der Menge an täglich zugeführten Kalorien und der Letalität besteht. Diese indirekte Korrelation hängt jedoch vom BMI ab. Signifikante Effekte finden sich nur bei Patienten mit einem BMI $<25$ bzw. $>35$. Für den Rest der Intensivpatienten (und das ist ja der größere Teil) scheint die exakte Höhe der Kalorienzufuhr keine so große prognostische Bedeutung zu besitzen. Somit sollte zumindest für diese zwei Gewichtsgruppen eine tägliche Zufuhr von 2000 kcal nicht unterschritten werden.

\section{Metabolisches Monitoring}

Unter künstlicher, speziell parenteraler Ernährungstherapie kann es zu einer Reihe von metabolischen Imbalanzen/ Nebenwirkungen kommen, zu deren Vorbeugung/Verhütung spezielle Überwachungsmaßnahmen angezeigt sind [16, 17].

Zum Monitoring der Fettutilisation ist es notwendig, in regelmäßigen Abständen die Plasmatriglyzeridkonzentrationen zu bestimmen. So lassen sich frühzeitig Fettverwertungsstörungen erkennen. Ferner erlaubt auch die mehrfach täglich durchgeführte Blutzuckerbestimmung (Blutzuckertagesprofil) den Nachweis von Kohlenhydratverwertungsstörungen. Die tägliche Bestimmung der Kreatinin- bzw. Harnstoffkonzentrationen kann nur sehr eingeschränkt als Hinweis für eine zunehmende Eiweißkatabolie bzw. Niereninsuffizienz herangezogen werden. Die Bestimmung dieser Serumkonzentrationen ist jedoch notwendig, um in Verbindung mit einem akuten Nierenversagen den Anstieg dieser harnpflichtigen Substanzen in den toxischen Bereich hinein frühzeitig erkennen und gegebenenfalls entsprechende therapeutische Maßnahmen einleiten zu können. Die Kenntnis der Kreatinin-Clearance ist unter anderem auch für eine korrekte Medikamentendosierung bedeutsam. Die Bestim- 
Tab. 4. Metabolisches Monitoring (Mindeststandard) für chirurgische Intensivpatienten mit künstlicher Ernährung (enteral und parenteral) $)^{\mathrm{a}}$

\begin{tabular}{|c|c|}
\hline Laborparameter & Häufigkeit der Bestimmung \\
\hline $\mathrm{Na}$ & mindestens 1-mal pro Schicht (BGA) \\
\hline $\mathrm{K}$ & mindestens 2-mal pro Schicht (BGA) \\
\hline $\mathrm{Ca}$ & $\begin{array}{l}\text { 2-mal pro Woche } \\
\text { nach jeder Substitution } \\
\text { nach Thyreoidektomie } \\
\text { täglich bei folgenden Patienten: Hyperparathyreoidismus mit und ohne } \\
\text { OP, Nierenersatztherapie mit Citrat-Antikoagulation }\end{array}$ \\
\hline Ca-ionisiert & $\begin{array}{l}\text { mindestens 1-mal pro Schicht (BGA) } \\
\text { mindestens 2-mal pro Schicht bei Citrat-Dialyse oder SLEDD mit Citrat }\end{array}$ \\
\hline Kreatinin & $\begin{array}{l}\text { täglich in der Akutphase der Erkrankung } \\
\text { bei stabilen Patienten mit Langzeiternährung 2-mal pro Woche } \\
\text { nach jeder Dialyse/SLEDD-Behandlung (Qualitätskontrolle) }\end{array}$ \\
\hline Harnstoff & $\begin{array}{l}\text { 2-mal pro Woche } \\
\text { bei erhöhtem Kreatinin oder erniedrigter Kreatinin-Clearance und bei } \\
\text { Nierenersatztherapie täglich } \\
\text { nach jeder Dialyse/SLEDD-Behandlung (Qualitätskontrolle) }\end{array}$ \\
\hline Kreatinin-Clearance & $\begin{array}{l}\text { nach Bedarf bei Verdacht auf/sich entwickelnde/bestehende } \\
\text { Niereninsuffizienz }\end{array}$ \\
\hline Se-Albumin & 2-mal pro Woche \\
\hline Magnesium & $\begin{array}{l}\text { mindestens 2-mal pro Woche } \\
\text { mindestens 2-mal pro Schicht bei Citrat-Dialyse oder SLEDD mit Citrat } \\
\text { nach jeder Substitution }\end{array}$ \\
\hline Phosphat & mindestens 2-mal pro Woche nach jeder Substitution \\
\hline Cholesterin & 2-mal pro Woche \\
\hline Triglyzeride & 2-mal pro Woche \\
\hline Selen & keine Routinebestimmung \\
\hline Zink & keine Routinebestimmung \\
\hline Eisen & keine Routinebestimmung \\
\hline \multicolumn{2}{|c|}{$\begin{array}{l}{ }^{a} \text { Die Tabelle zeigt den Standard der Chirurgischen Intensivstation am UKSH, Campus Lübeck. } \\
\text { Er erhebt keinen Anspruch auf Allgemeingültigkeit. } \\
\text { BGA = Blutgasanalyse. }\end{array}$} \\
\hline
\end{tabular}

mung mit einem 12-h-Sammelurin hat eine ausreichende Genauigkeit.

Der Elektrolytbedarf des Patienten wird üblicherweise durch tägliche Bestimmung entsprechender Serumkonzentrationen abgeschätzt. Besonders hervorzuheben sind Magnesium und Kalium, da diese Elektrolyte insbesondere beim Auslösen von Herzrhythmusstörungen eine wichtige Rolle spielen. Auch Kalzium, Phosphat, Chlorid und Natrium sollten überwacht werden, um Einschränkungen der Muskelfunktionen, Störungen der Wundheilung, aber auch metabolische Alkalosen frühzeitig erkennen zu können. Die Intensität der Überwachung richtet sich dabei nach der Schwere des Krankheitsbildes.

Auch beim metabolischen Monitoring ist es sinnvoll, über Regeln für Routine-Laborbestimmungen während künstlicher Ernährung zu verfügen [16]. In Tabelle 4 ist der Standard für das metabolische Monitoring einer chirurgischen Intensivstation dargestellt, in dem das Minimum der Labor- kontrollen festgelegt ist. Dabei hängt die Häufigkeit der Bestimmungen von der Erkrankungsschwere und den klinischen Veränderungen beim Patienten ab.

\section{Weiterführende Internetadressen}

- www.pharmatrix.de: Für Informationen/Datenbank Pharmaka.

- www.dgem.de: Internetpräsenz der Deutschen Gesellschaft für Ernährungsmedizin (mit deutschsprachigen Guidelines).

- www.espen.org: Internetseite der European Society for Clinical Nutrition and Metabolism (Leitlinien).

- www.uni-duesseldorf/AWMF: AWMF-online, Leitlinien der Arbeitsgemeinschaft der Wisssenschaftlichen Medizinischen Fachgesellschaften.

\section{Disclosure Statement}

Es bestehen keine Interessenkonflikte. 


\section{Literatur}

1 Hartl WH, Rittler P: Veränderungen des Substratstoffwechsels bei chirurgischen Erkrankungen unter besonderer Berücksichtigung des Eiweißhaushalts. Akt Ernährungsmed 1997;22:154-163.

2 Hartl WH, Inthorn D: Postoperative Systemkomplikationen; in Bruch H-P, Trentz O (Hrsg): Chirurgie. München, Urban \& Fischer, 2000, pp 223-242.

3 Hartl WH, Rittler P: Perioperative Infusionstherapie - Prinzipien. Chirurg 2002;73:1067-1086.

4 Hartl WH, Rittler P, Jauch K-W: XI-3. Metabolische und endokrine Besonderheiten beim operativen Intensivpatienten - Konsequenzen für die Therapie; in Eckart J, Forst H, Buchardi H (Hrsg): Intensivmedizin. Landsberg, ecomed, 2003, pp 1-28.

5 Hartl WH, Jauch K-W: Blutzucker in der Intensivmedizin. Akt Ernährungsmed 2006;31(suppl 1): S81-S88.

6 Alberda C, Gramlich L, Jones N, Jeejeebhoy K, Day AG, Dhaliwal R, Heyland DK: The relationship between nutritional intake and clinical outcomes in critically ill patients: results of an international multicenter observational study. Intensive Care Med 2009;35:1728-1737.

7 Kreymann G, Adolph M, Mueller MJ; Working group for developing the guidelines for parenteral nutrition of The German Association for Nutritional Medicine: Energy expenditure and energy intake - Guidelines on Parenteral Nutrition, Chapter 3. Ger Med Sci 2009;7:Doc25.
8 Bankhead R, Boullata J, Brantley S, Corkins M, Guenter P, Krenitsky J, Lyman B, Metheny NA, Mueller C, Robbins S, Wessel J; A.S.P.E.N. Board of Directors: Enteral nutrition practice recommendations. JPEN J Parenter Enteral Nutr 2009;33: 122-167.

9 Hartl WH, Rittler P, Jauch K-W: Gefahren der Immunonutrition bei Sepsis. Intensiv- und Notfallbehandlung 2005;30:256-264.

10 Stein J, Boehles HJ, Blumenstein I, Goeters C, Schulz R; Working group for developing the guidelines for parenteral nutrition of The German Association for Nutritional Medicine: Amino acids Guidelines on Parenteral Nutrition, Chapter 4. Ger Med Sci 2009;7:Doc24.

11 Bolder U, Ebener C, Hauner H, Jauch KW, Kreymann G, Ockenga J, Traeger K: Working group for developing the guidelines for parenteral nutrition of The German Association for Nutritional Medicine. Carbohydrates - Guidelines on Parenteral Nutrition, Chapter 5. Ger Med Sci 2009;7:Doc23.

12 Brown RO, Compher C; American Society for Parenteral and Enteral Nutrition Board of Directors: A.S.P.E.N. clinical guidelines: nutrition support in adult acute and chronic renal failure. JPEN J Parenter Enteral Nutr 2010;34:366-377.
13 Druml W, Kierdorf HP; Working group for developing the guidelines for parenteral nutrition of The German Association for Nutritional Medicine: Parenteral nutrition in patients with renal failure Guidelines on Parenteral Nutrition, Chapter 17. Ger Med Sci 2009;7:Doc11.

14 Biesalski HK, Bischoff SC, Boehles HJ, Muehlhoefer A; Working group for developing the guidelines for parenteral nutrition of The German Association for Nutritional Medicine: Water, electrolytes, vitamins and trace elements - Guidelines on Parenteral Nutrition, Chapter 7. Ger Med Sci 2009; 7:Doc21.

15 Rittler P, Bolder U, Hartl WH, Jauch KW: Enterale Ernährung: Indikation und Zugangswege. Chirurg 2006;77:1063-1078.

16 Hartl WH, Rittler P: Metabolisches Monitoring des Intensivpatienten. Akt Ernährungsmed 2002;27: 408-415.

17 Hartl WH, Jauch KW, Parhofer K, Rittler P, Working group for developing the guidelines for parenteral nutrition of The German Association for Nutritional Medicine: Complications and monitoring - Guidelines on Parenteral Nutrition, Chapter 11. Ger Med Sci 2009;7:Doc17. 